Check for updates

Cite this: RSC Adv., 2018, 8, 5816

Received 12th December 2017

Accepted 26th January 2018

DOI: $10.1039 / c 7 r a 13276 j$

rsc.li/rsc-advances

\section{Comparison of two online extraction systems and development of the online SPE-HPLC-DAD method to simultaneously determine ten $\beta$-amino alcohol drugs in plasma $\uparrow$}

\begin{abstract}
Man Wang, (DD ab Lei Liu, ${ }^{a}$ Zheng Yin ${ }^{\mathrm{ab}}$ and Yaxin Lu*ab
For the chromatographic analysis of biological samples, sample preparation requires efficient matrix removal and retention of the analytes. The development of online pretreatment technologies provides a fully automated solution for biological sample analysis. Online pretreatment solutions improve both throughput and precision. In this study, we compared two online extraction systems, an online solid-phase extraction (SPE) system and an online turbulent flow chromatography (TFC) extraction system, which are being applied by more analysts at present. The comparison showed that the TFC extraction produced better matrix removal effects, while, peak analysis showed that the online SPE system had obvious advantages in peak shape and efficiency. Thus, we developed an automated online SPE-high performance liquid chromatography (HPLC)-diode array detector (DAD) method for the simultaneous determination of ten $\beta$ amino alcohols. The results provide a reference for analysts to choose an appropriate online pretreatment method and provide a solution for biological sample analysis of $\beta$-amino alcohol drugs.
\end{abstract}

\section{Introduction}

Due to the complex matrix in biological samples, matrix removal and analyte extraction are always important for chromatographic analysis. Conventional extraction techniques mainly include protein precipitation (PPT), ${ }^{1}$ liquid-liquid extraction (LLE) ${ }^{2}$ and off-line SPE methods. ${ }^{3}$ However, these manual operations are prone to human error and are timeconsuming, which significantly affects the throughput and reproducibility of the process. ${ }^{4}$ Sample pretreatment is the labor-intensive, time-limiting step in bioanalytical processes, typically taking $80 \%$ of the total analysis time and involving intensive manual processing. ${ }^{5}$

An online preparation method, such as direct detection using probe electrospray ionization mass spectrometry ${ }^{6-8}$ and online extraction methods, could overcome the shortcomings mentioned above to a great extent. The most commonly used online extraction methods are based on solid phase extraction $^{9-12}$ and turbulent flow chromatography. ${ }^{13-15}$ The fully automated online SPE-HPLC technique, which instrumentally integrates the biological fluids clean-up process with bioanalysis, has been widely applied to remove interfering matrix

${ }^{a}$ College of Pharmacy, Nankai University, Tianjin 300071, PR China. E-mail: yaxinlu@ nankai.edu.cn

${ }^{b}$ State Key Laboratory of Medicinal Chemical Biology, Nankai University, Tianjin 300071, PR China

$\dagger$ Electronic supplementary information (ESI) available. See DOI: 10.1039/c7ra13276j substances. Turbulent flow chromatography (TFC) was introduced in the late 1990s as a technique for direct injection of biological fluids into a column. ${ }^{16}$ Under turbulent flow conditions, small molecules in solution diffuse more extensively into the pores of particles than large molecules, leading to the separation of substances by size. ${ }^{\mathbf{1 7}}$ With the advantage of automation, this technique has been used for high throughput sample preparation to detect various analytes in complex samples. ${ }^{18-20}$

Both of these online preparation methods are increasingly being used for biological sample analysis, but no comparative analysis of the two online pretreatment systems is available to provide a reference to aid analysts' selection. Hence, we evaluated two different online systems, Turboflow and online SPE, for the extraction of samples and comparied several parameters such as matrix removal effects and peak analysis. To obtain a more representative result, we chose a series of $\beta$-amino alcohols with different polarities as the target analytes. After comparison, we selected the online SPE-HPLC-DAD system for method validation. Our lab has previously reported an HPLCmass spectrometry method for the simultaneous determination of a broad range of cardiovascular drugs in plasma. ${ }^{21}$ In this study, we made a few modifications to the compounds selection and detector, adding a more polar compound, adrenaline, which increased the difficulty of online extraction and simultaneous detection, and replacing mass spectrometry was with DAD for more convenient application, as DAD is a cheaper detector, which is still widely used in hospitals in China. We 
provide a DAD method enabling laboratories and hospitals without MS to conduct biological sample analysis.

\section{Experimental}

\section{Reagents and materials}

Reference standards of adrenaline, salbutamol, timolol, carteolol, ephedrine, mexiletine, clorprenaline, clenbuterol, propranolol and carvedilol were purchased from the National Institutes of Food and Drug Control (Beijing, China). Their chemical structures and physicochemical parameters are shown in Fig. 1. Analytical purity sodium dihydrogen phosphate and hydrochloric acid were obtained from Tianjin Chemical Reagents Company (Tianjin, China). HPLC grade acetonitrile (ACN) and water were obtained from Tedia Company, Inc. (Fairfield, USA).

\section{Plasma}

Drug-free heparinized rat plasma was collected from male Sprague-Dawley rats (body weight: $220-250 \mathrm{~g}$ ) obtained from the Laboratory Animal Center, Academy of Military Medical Science (Beijing, China). The animal facilities and protocols were approved by the Institutional Animal Care and Use Committee of Nankai University. All procedures were carried out in accordance with the Guidelines for Animal Experimentation of Nankai University (Tianjin, China).

\section{Preparation of calibration work solutions and quality control samples}

The stock solutions of all drugs were prepared at a concentration of $1.00 \mathrm{mg} \mathrm{mL}^{-1}$ with ACN/water $(1: 1, \mathrm{v} / \mathrm{v})$ and stored at $4{ }^{\circ} \mathrm{C}$. The solutions of standards and quality controls were diluted with blank plasma $(10: 90, \mathrm{v} / \mathrm{v})$ to prepare a series of samples with different concentrations ranging from $10 \mathrm{ng} \mathrm{mL}^{-1}$ to $2560 \mathrm{ng} \mathrm{mL}^{-1}$. Quality control (QC) samples including solutions for the lower limit of quantification (LLOQ), limit of detection (LOD), QC low (QCL), QC middle (QCM) and QC high (QCH) of 40, 160 and $1280 \mathrm{ng} \mathrm{\textrm {mL } ^ { - 1 }}$ were prepared for validating the method. All of the work solutions were freshly diluted when used.

\section{Online SPE-HPLC method}

Online SPE and HPLC analysis were carried out using an UltiMate 3000 Dual-Gradient HPLC system (Sunnyvale, CA, USA) equipped with two ternary pumps, a vacuum degasser, an autosampler and a thermostatted column compartment with a six-way valve. The online SPE column was a CAPCELL PAK MF Ph-1 column $(4.0 \times 10 \mathrm{~mm}$, Shiseido, Japan $)$ and the analytical column was a Venusil MP C18 column $(5 \mu \mathrm{m}, 4.6 \times 150 \mathrm{~mm}$, Agela Technologies, China).

The online pretreatment and separation were accomplished by three steps, including a loading phase, a washing phase and an elution phase, as reported before. ${ }^{22}$ The loading and washing time was $1 \mathrm{~min}$, and the elution time was $9 \mathrm{~min}$ at a flow rate of $1 \mathrm{~mL} \min ^{-1}$. The mobile phases and gradient are shown in Fig. 2 and Table S1.†

\section{Online TFC-HPLC method}

For the online TFC-HPLC method, the same instruments were used as in the online SPE-HPLC method, except the SPE column was replaced by a TurboFlow HTLC C18-P XL column $(1.0 \times$ $5.0 \mathrm{~mm}$, Thermo Scientific, USA). The flow rate was increased<smiles>CNCC(O)c1ccc(O)c(O)c1</smiles><smiles>CN[C@H](C)[C@H](O)c1ccccc1</smiles><smiles>CC(C)(CCl)NCC(O)COc1cccc2c1CCC(=O)N2</smiles>

Adrenalin

(No.1, $\log P=0.28$ )

Salbutamol (No.2, $\log P=0.97$ )

\section{Ephedrine} (No.3, $\log P=0.93$ )
Carteolol

(No.4, $\log \mathrm{P}=1$ )<smiles>CC(Cl)NCC(O)c1ccccc1Cl</smiles>

Clorprenaline hydrochloride (No.5, $\log P=2.28$ )<smiles>Cc1cccc(C)c1OCC(N)Cl</smiles>

Mexiletine hydrochloride (No.8, $\log P=2.3$ )<smiles>CC(C)(C)NCC(O)COc1nsnc1N1CCOCC1</smiles>

Timolol hydrogenmaleate (No.6, $\log P=2.34$ )<smiles>CC(Cl)NCC(O)COc1cccc2ccccc12</smiles>

Propranolol Hydrochloride (No.9, $\log P=3.35$ )<smiles>CC(C)(C)NCC(O)c1cc(Cl)c(N)c(Cl)c1</smiles>

Clenbuterol (No.7, $\log P=2.25$ )<smiles>[R16][R6]([Y6])([O-])[N+]#N</smiles>

Carvedilol

(No.10, $\log P=3.12$ )

Fig. 1 The structures and $\log P$ of $\beta$-amino alcohols. 

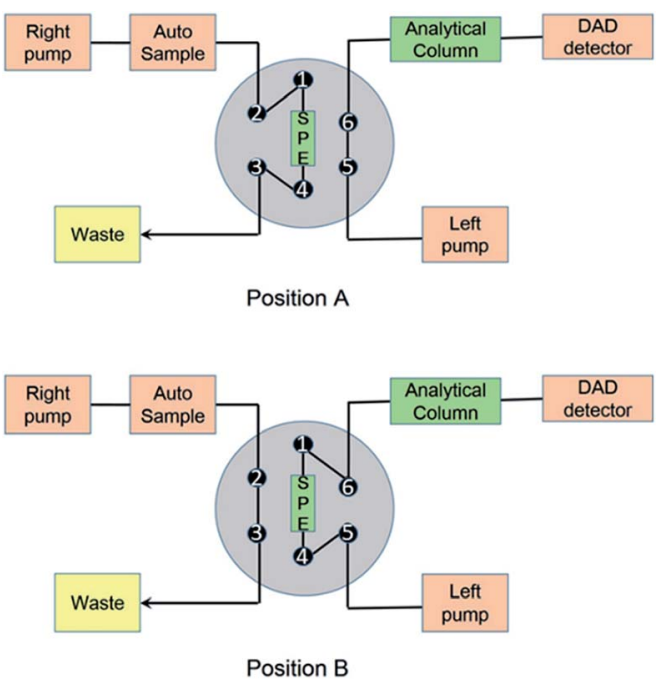

Fig. 2 Schematic diagram of the online SPE/TFC-HPLC-DAD system. ${ }^{22}$

for TFC extraction and the details are shown in Fig. 2 and Table S2.†

\section{Statistical analysis}

We conducted a $t$-test with Microsoft Excel, setting the conditions for a one-tailed, paired test. When the $p$ value $<0.05$, the difference was considered significant.

\section{Results and discussions}

\section{The comparison of the two pretreatment systems}

Retention ability. We compared the retention abilities of the two online analytical systems for ten compounds of different polarities. The results showed that all of the drugs could be extracted by the MF Ph-1 column in the online SPE system, while the TurboFlow C18P column could not retain the most polar compound, adrenaline. We speculated that the TurboFow $\mathrm{C} 18 \mathrm{P}$ column was less competent than the MF Ph-1 column in retaining strongly polar compounds with a $\log P \leq 0.28$.

The matrix removal effect. The matrix removal effect of pretreatment influences the service life of the analytical column, and the matrix peaks interfere with the analysis of the compounds. Hence, we compared the matrix removal effect of the two pretreatment systems. As the flow rate affects the formation of turbulent flow and mass transfer in TurboFlow columns, we analyzed the matrix residue at different loading rates of $2 \mathrm{~mL} \mathrm{~min}^{-1}, 3 \mathrm{~mL} \min ^{-1}$ and $4 \mathrm{~mL} \mathrm{~min}^{-1}$. We found that the TurboFlow $\mathrm{C} 18 \mathrm{P}$ column was more effective at a flow rate of

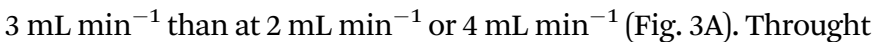
the comparison of two online pretreatment systems, we found that TurboFlow C18P column showed a greater ability to remove the matrix (Fig. 3B). The advantage of TurboFlow columns in removing matrix may duo to their separation principle. During the extraction and separation process, macromolecules do not have time to diffuse into the pores and are more easily flashed through the column by the high-velocity mobile phase. ${ }^{17}$
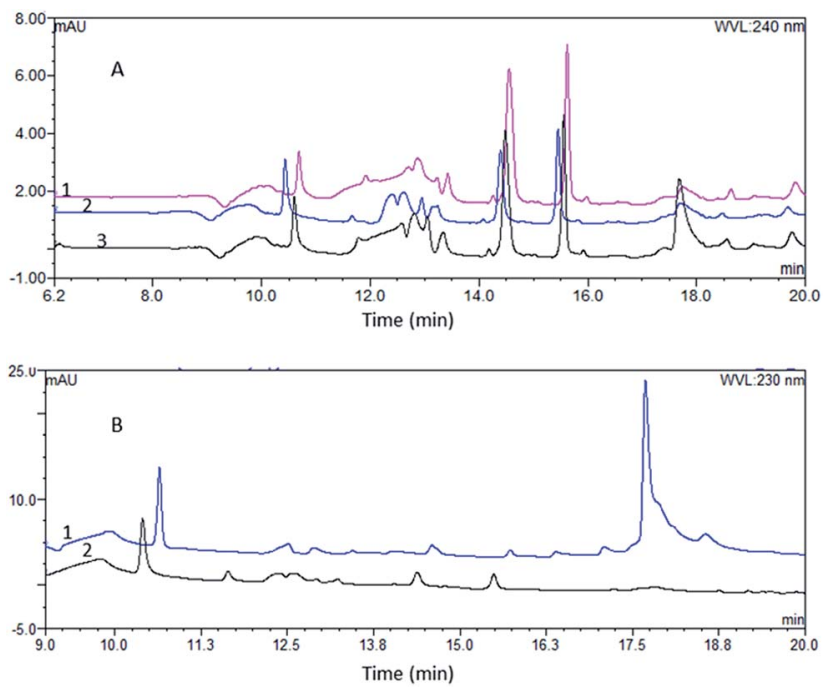

Fig. 3 The chromatograms of blank plasmas after pretreatment (A): the chromatogram of blank plasma after pretreatment obtained from the Turboflow C18P column at flow rate of $2 \mathrm{~mL} \mathrm{\textrm {min } ^ { - 1 } ( 1 ) , 3 \mathrm { mL } \mathrm { min }} \mathrm{m}^{-1}$ (2), $4 \mathrm{~mL} \mathrm{~min} \mathrm{~min}^{-1}$ (3); (B): the chromatogram of blank plasma after analysis by the online SPE HPLC system (1) and the online TFC-HPLC system (2).

Peak analysis. Comparison of peak asymmetry, resolution, and theoretical plate numbers helps in understanding the differences in peak shape and efficiency of the two different online pretreatment systems. We selected the drugs numbered $2,3,4,5,6,7$, and 8 that were detected at the same wavelength to compare the resolution. The resolution $(R)$ was calculated as follows: ${ }^{23}$

$$
R=2\left(t_{\mathrm{R} 2}-t_{\mathrm{R} 1}\right) /\left(W_{1}+W_{2}\right)
$$

where $t_{\mathrm{R} 2}$ is the retention time of the last peak in two adjacent peaks, $t_{\mathrm{R} 1}$ is the retention time of the first peak in two adjacent peaks, and $W_{1}$ and $W_{2}$ are the peak widths of two adjacent peaks. As shown in Fig. 4A, the resolutions of 5 and 7 were close in both systems, while the other five drugs all showed better resolutions in the online SPE-HPLC system.

To characterize the peak asymmetry, the tailing factor $(T)$ was calculated as below: ${ }^{23}$

$$
T=W_{0.05 h} / 2 d_{1}
$$

where $W_{0.05 h}$ is the peak width at 5\% of the peak maximum and $d_{1}$ is the distance between the peak apex and the peak front. The results of the peak symmetry comparison are shown in Fig. 4B. Statistical results showed that the peak symmetry differences between the two systems for 2 and 6 were not significant, while the peak shapes of the other seven drugs were more symmetrical in the online SPE system.

The number of theoretical plates is an important parameter for evaluating the separation performance of a chromatographic system. As the same analytical column was used for both, the comparison of theoretical plates could reflect the efficiency of the two different pretreatment systems. The theoretical plates $(n)$ were calculated as below: ${ }^{23}$ 

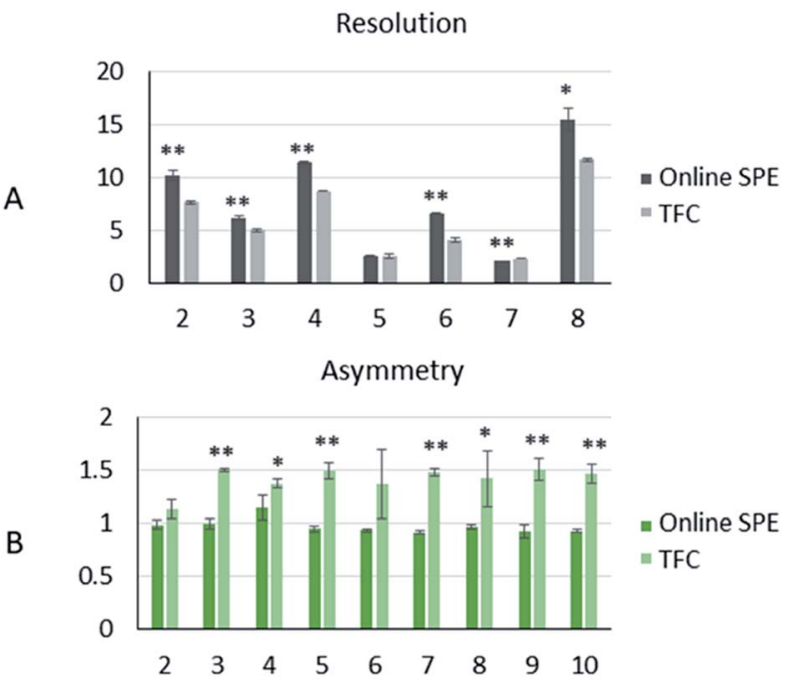

Theoretical plates

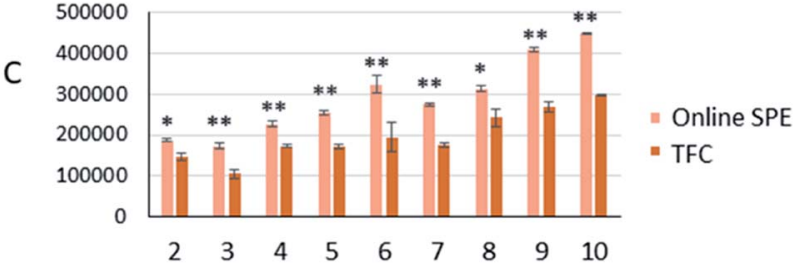

Fig. 4 The peak analysis of 9 drugs produced from the two online analytical systems (A): the comparison of resolutions in the two online extraction systems; $(B)$ : the comparison of the tailing factors of nine drugs in the two online extraction systems; (C): the comparison of theoretical plates in the two online extraction systems. "**" means $p<$ 0.01 , "*" means $p<0.05$.

$$
n=16\left(t_{\mathrm{R}} / W\right)^{2}
$$

where $t_{\mathrm{R}}$ is retention time and $W$ is peak width. From this calculation, all drugs showed more theoretical plates in the online SPE system (Fig. 4C). Summarizing the results of the peak analysis, we could conclude that the online SPE extraction system had significant advantages in terms of separation efficiency and peak symmetry.

\section{Method validation}

Based on the comparison of retention abilities and peak analysis, we performed a method validation of the online SPE-HPLCDAD method.

Selectivity. The selectivity was studied by injecting QC plasma samples of the drugs using the developed method. The chromatograms of the drugs at the detection wavelengths were compared with the chromatograms of the blank plasma. The results showed little interference of endogenous proteins or matrix, indicating good selectivity (shown in Fig. 5).

Recovery. The extraction recoveries (RE) were assessed according to the procedure described by Matuszewski et al. ${ }^{24}$ The set 1 samples (pre-extraction spiked matrix) were prepared using blank plasma spiked with different levels of QC sample solutions (9:1, v/v) for online SPE HPLC-DAD

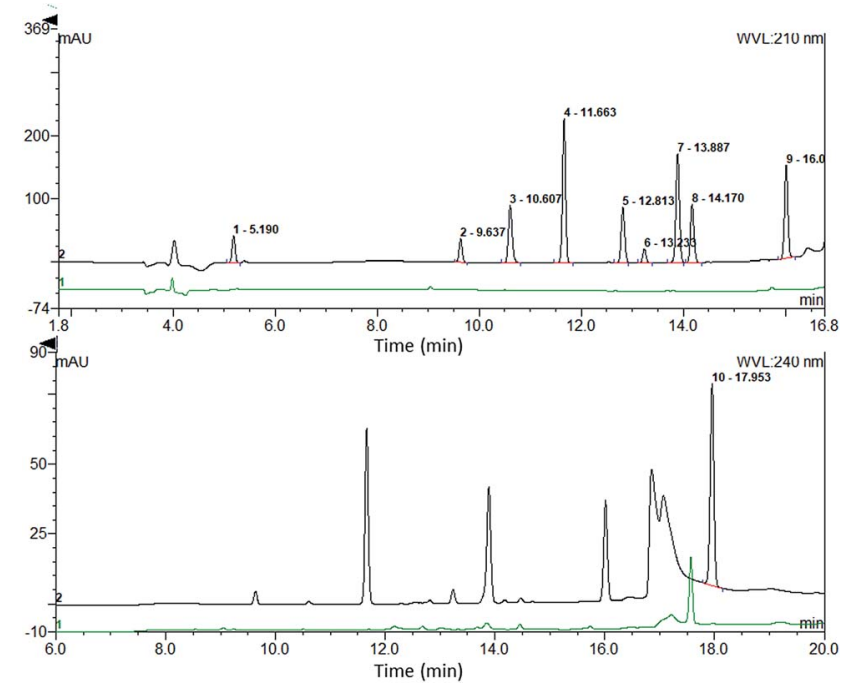

Fig. 5 Comparison of the analyte chromatogram with the blank plasma chromatogram.

analysis. The set 2 samples were prepared using the mobile phase containing different QC solution levels. The set 2 samples were analyzed directly by HPLC-DAD. The extraction recoveries were determined by comparing the mean response of the set 1 samples to that of the set 2 samples at each QC level. The extraction recoveries of the QC samples are presented in Table S3. $\dagger$ According to the guidance of the U.S. Food and Drug Administration, the recovery of the analyte should be precise and reproducible. ${ }^{25}$ The extraction recoveries at different concentrations ranged from $92.38 \%$ to $98.53 \%$ and the RSDs of the recoveries ranged from $3.43 \%$ to $5.79 \%$.

LLOQ and linearity. Decreasing concentrations of the analytes were injected into the analytical system to determine the minimal concentration with a signal-to-noise ratio $(\mathrm{S} / \mathrm{N})$ of LLOQ at least $5: 1$, adequate precision with a coefficient of variation (CV) less than $20 \%$ and trueness within $20 \%$ of the nominal value (i.e., trueness between 80 and 120\%) for each analysis run. ${ }^{18}$ The LOD is the minimum concentration of the sample at which the $\mathrm{S} / \mathrm{N}$ is not less than 3. The LLOQ and LOD of each drug is shown in Table 1. The standard calibration curves were constructed using the peak area of each analyte versus the nominal concentrations of the eight plasma standards. Five standard curves were conducted for each drug in parallel. Linear least-square regression analysis, with a weighting factor of $1 / x^{2}$, was performed to assess the linearity, as well as to generate the standard calibration equation: $y=a x+b$, where $y$ is the peak area, $x$ is the concentration, $a$ is the slope and $b$ is the intercept of the regression line. The method was linear over the concentration range of each analyte $(r>0.99)$. The mean values of the linear regression equation for each of the analytes are listed in Table 1.

Trueness and precision. The intra-day trueness and precision were evaluated by repeated analysis of the quality control (QC) samples at low, middle and high levels through five replicates performed on the same day, whereas the inter-day 
Table 1 LOD, LLOQ and regression data of the analytes $(n=5)$

\begin{tabular}{|c|c|c|c|c|c|c|c|c|c|}
\hline Analyte & LOD $\left(\mathrm{ng} \mathrm{mL}^{-1}\right)$ & $\mathrm{LOQ}\left(\mathrm{ng} \mathrm{mL}^{-1}\right)$ & $\begin{array}{l}\text { Linear range } \\
\left(\mathrm{ng} \mathrm{mL}^{-1}\right)\end{array}$ & \multicolumn{2}{|c|}{ Slope $\left(\times 10^{4}\right)$} & \multicolumn{2}{|c|}{ Intercept $\left(\times 10^{3}\right)$} & \multicolumn{2}{|l|}{$R^{2}$} \\
\hline 2 & 5 & 20 & $20-2560$ & 16.0 & 2.6 & 30.2 & 5.0 & 0.9960 & 0.0031 \\
\hline 3 & 2.5 & 10 & $10-2560$ & 51.7 & 2.9 & -23.4 & 6.1 & 0.9995 & 0.0007 \\
\hline 4 & 2.5 & 10 & $10-2560$ & 93.0 & 2.6 & -1.9 & 0.4 & 0.9999 & 0.0001 \\
\hline 7 & 5 & 20 & $20-2560$ & 93.3 & 3.2 & -193.8 & 20.1 & 0.9998 & 0.0001 \\
\hline 8 & 10 & 40 & $40-2560$ & 41.7 & 2.1 & 59.8 & 5.6 & 0.9994 & 0.0008 \\
\hline 9 & 10 & 40 & $40-2560$ & 85.3 & 5.1 & -273.6 & 50.6 & 0.9996 & 0.0002 \\
\hline 10 & 10 & 40 & $40-2560$ & 34.3 & 4.7 & 52.6 & 1.7 & 0.9992 & 0.0006 \\
\hline
\end{tabular}

trueness and precision were evaluated on three independent days. The trueness was expressed as a percentage of the measured value versus the nominal concentration, while the precision was assessed by the relative standard deviation (RSD) for sets of replicates. The acceptance criteria of data included a trueness within a $15 \%$ bias of the nominal values and a precision within $15 \%$ of the RSD. The intra-day and inter-day precisions and trueness value of the QC samples are presented in Table 2. The RSD values of the QC samples were in the range of $1.4-13.2 \%$, and the trueness was between $88.4 \%$ and $114.1 \%$. These results met the acceptance criteria for trueness $(85-115 \%)$ and precision $(<15 \%)$ and thus allowed for an accurate assessment of the analytes in plasma. ${ }^{24}$

Table 2 The trueness and precision of all drugs $(n=5)$

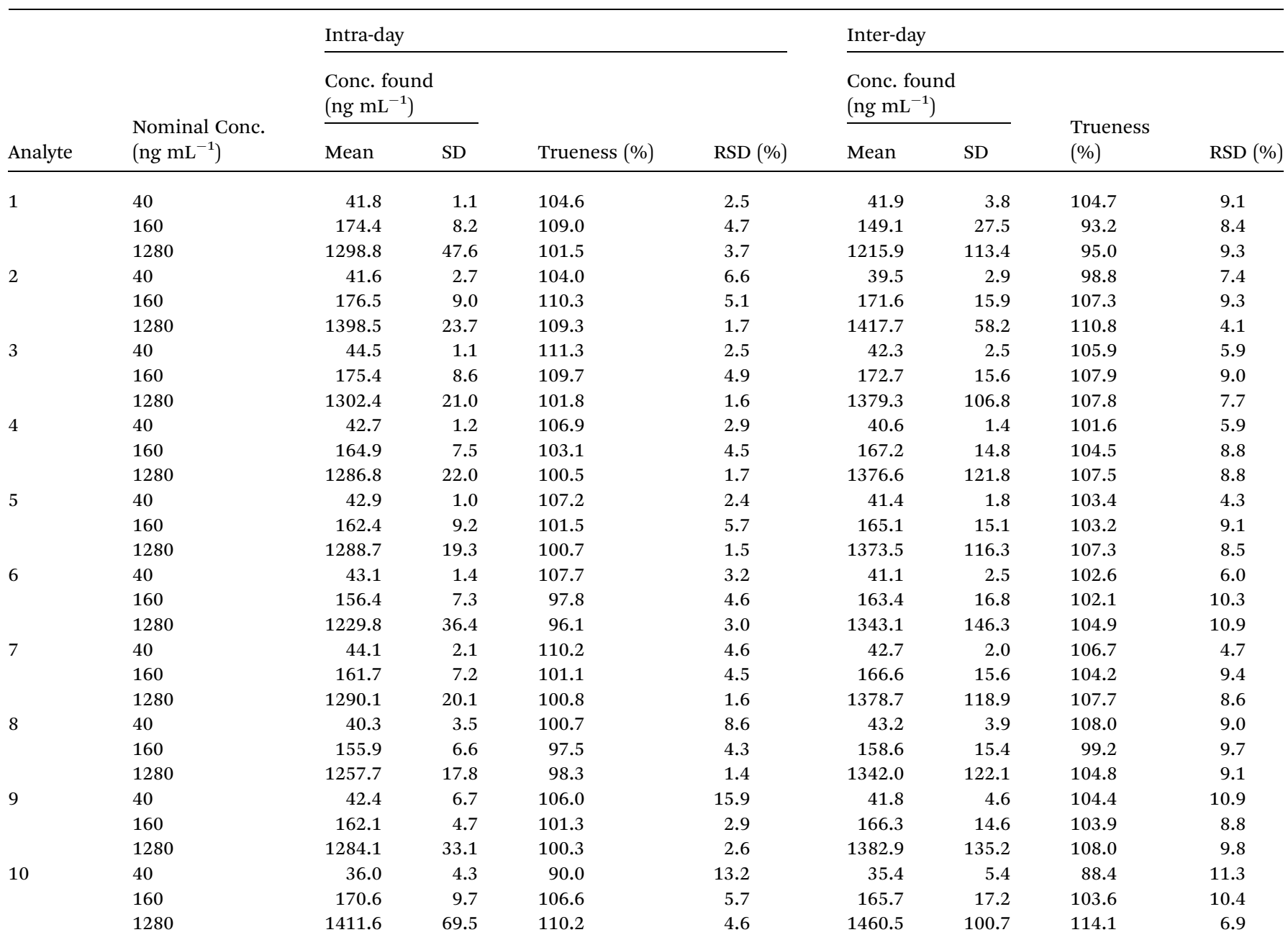




\section{Conclusions}

Using a series of plasma samples of drugs with different polarities, we conducted a multi-aspect comparison of two online extraction systems. From the results, the online TFC extraction system showed better matrix removal effects. Thus, it may be a more appropriate option for detectors sensitive to matrix interference, such as mass spectrometry. The comparison of retention ability and peak analysis revealed that the online SPE extraction system had significant advantages in retaining more polar compounds and in separation efficiency, as well as in peak shape. These advantages helped improve the signals of drugs and the limit of quantification. Therefore, the online SPE extraction system was more suitable for the DAD detector, whose sensitivity was worse than that of mass spectrum. Finally, we established an online SPE-HPLC-DAD method for simultaneous quantitation of ten $\beta$-amino alcohols and performed method validation. Compared to mass spectrum, the DAD detector was easier to use and had a wider quantitative linear range than mass spectrum. ${ }^{21}$ In conclusion, our comparison of the two online pretreatment systems provides researchers a reference to choose the appropriate method based on the analyte polarity and detector used. The bioanalytical method for simultaneous determination of ten $\beta$-amino alcohols may provide a solution for therapeutic drug monitoring.

\section{Conflicts of interest}

There are no conflicts to declare.

\section{Acknowledgements}

This work was supported by the National Basic Research Program of China (973 program, Grant No. 2013CB911104, 2013CB911100), the National Natural Science Foundation of China (Grant No. 21672115, 21572116), the "111" Project of the Ministry of Education of China (Project No. B06005), and the Natural Science Foundation of Tianjin (Project No. 17JCYBJC28800).

\section{References}

1 J. He, W.-b. Li and C.-j. Ruan, Acta Pharmacol. Sin., 2013, 34, 140-141.

2 A. Mustafa and C. Turner, Anal. Chim. Acta, 2011, 703, 8-18.

3 S. C. Cobzac and S. Gocan, J. Liq. Chromatogr. Relat. Technol., 2011, 34, 1157-1267.

4 W. Xie, C. M. Chavez-Eng, W. Fang, M. L. Constanzer, B. K. Matuszewski, W. M. Mullett and J. Pawliszyn, J.
Chromatogr. B: Anal. Technol. Biomed. Life Sci., 2011, 879, 1457-1466.

5 H. Ktaoka and K. Saito, J. Pharm. Biomed. Anal., 2011, 54, 926-950.

6 S. Saha, L. C. Chen, M. K. Mandal and K. Hiraoka, J. Am. Soc. Mass Spectrom., 2013, 24, 341-347.

7 S. Saha, M. K. Mandal, L. C. Chen, S. Ninomiya, Y. Shida and K. Hiraoka, Mass Spectrosc., 2013, 2, S0008.

8 S. Saha, M. K. Mandal, H. Nonami and K. Hiraoka, Anal. Chim. Acta, 2014, 839, 1-7.

9 L. Liu, K. N. Liu, Y. B. Wen, H. W. Zhang, Y. X. Lu and Z. Yin, J. Chromatogr. B: Anal. Technol. Biomed. Life Sci., 2012, 893, 21-28.

10 Z. León, A. Chisvert, A. Balaguer and A. Salvador, Anal. Chim. Acta, 2010, 664, 178-184.

11 J. R. Griffiths, S. Perkins, Y. Connolly, L. Zhang, M. Holland, V. Barattini, L. Pereira, A. Edge, H. Ritchie and D. L. Smith, J. Chromatogr. A, 2012, 1232, 276-280.

12 J. M. Zhang, W. S. Lin, X. N. Li, N. Yu, X. M. Ling, G. Fu, R. T. Li and J. R. Cui, J. Sep. Sci., 2012, 35, 721-725.

13 C. Chassaing, H. Stafford, J. Luckwell, A. Wright and A. Edgington, Chromatographia, 2005, 62, 17-24.

14 D. Zimmer, V. Pickard, W. Czembor and C. Muller, J. Chromatogr. A, 1999, 854, 23-35.

15 L. Couchman, Biomed. Chromatogr., 2012, 26, 892-905.

16 H. M. Quinn and J. J. Takarewski, Int. Pat., WO97/16724, 1997.

17 P. J. Rudewicz, Bioanalysis, 2011, 3, 1663-1671.

18 D. Molins-Delgado, M. D. M. Olmo-Campos, G. Valeta-Juan, V. Pleguezuelos-Hernandez, D. Barcelo and M. S. Diaz-Cruz, Environ. Res., 2018, 161, 532-539.

19 M. Lopez-Garcia, R. Romero-Gonzalez, M. Lacasana and A. Garrido Frenich, J. Pharm. Biomed. Anal., 2017, 146, 378386.

20 H. D. Shin, J. H. Suh, J. Kim, H.-D. Cho, S. D. Lee, K. S. Han, Y. Wang and S. B. Han, J. Pharm. Biomed. Anal., 2017, 145, 46-51.

21 L. Liu, Y. B. Wen, K. N. Liu, L. Sun, Y. X. Lu and Z. Yin, $R S C$ Adv., 2014, 4, 19629-19639.

22 M. Wang, Y.-B. Wen, K. N. Liu, G. Si, L. Liu, Z. Yin and Y. X. Lu, Chin. J. Anal. Chem., 2014, 42, 1729-1733.

23 Chinese Pharmacopoeia CommissionChinese Pharmacopoeua (two), 2010, appendix V, pp. 29-31.

24 B. K. Matuszewski, M. L. Constanzer and C. M. Chavez-Eng, Anal. Chem., 2003, 75, 3019-3030.

25 https://www.fda.gov/downloads/Drugs/ GuidanceComplianceRegulatoryInformation/Guidances/ UCM070107.pdf. 